

\section{Factores de éxito y fracaso en la implementacion de proyectos con inversión del fondo de la estrategia de reducción de la pobreza en los municipios de Intibucá y San Isidro}

José Ramón Coto García*

EdtTH Yolany Zelaya**

RESUMEN. La investigación sobre factores de éxito y fracaso en el desarrollo de proyectos financiados con fondos de la estrategia de ERP, conduce a reflexionar respecto a las diferentes experiencias que han tenido los municipios de Intibucá y San Isidro ubicados en el departamento de Intibucá.

De manera general un factor de éxito ha sido la anuencia de las autoridades municipales en coordinar con otros actores la formulación e implementación de proyectos. Otro es la puesta en práctica de instrumentos normativos como ser la Ley de contratación del Estado, en el sentido de hacer las compras por medio de licitación pública para demostrar transparencia en el proceso y obtener los mejores precios de insumos. Sin embargo, existen factores de fracaso como dificultades de monitoreo efectivo tanto en la formulación como en la ejecución de proyectos, falta de recurso humano por parte de las municipalidades, la inexistencia de organismos de control social como las comisiones de trasparencia y la enorme demanda de recursos lo que dificulta beneficiar todas las comunidades del municipio.

Palabras clave: pobreza, proyectos, estrategia.

\section{INTRODUCCIÓN}

Honduras es uno de los 189 países que participaron en la Cumbre del Milenio celebrada en Nueva York en el mes de septiembre del año 2000, entre los acuerdos de la misma se destacan los ocho Objetivos de Desarrollo del Milenio (ODMs).

Tomando como referencia dicho marco, la Universidad Nacional Autónoma de Honduras (UNAH), por medio de la Maestría en Demografía Social; ha diseñado e impartido el Diplomado en Objetivos de Desarrollo del Milenio con Énfasis en Análisis Sociodemográfico, considerando que el logro de las ODMs, es una responsabilidad compartida, en tal sentido se ha desarrollado una investigación sobre el impacto de la inversión de los fondos de la Estrategia de Reducción de la Pobreza en los municipios de San Isidro e Intibucá, ubicados en el departamento de Intibucá.

Los Objetivos de Desarrollo del Milenio son parámetros o metas que nos sirven de base de comparación de cómo estamos y dónde queremos estar. Esta investigación se basa en el objetivo 1: Erradicar la Pobreza Extrema y el Hambre. De manera general el término pobreza significa carencia de condiciones y oportunidades básicas. Una vida digna es no carecer de una vivienda, un trabajo, ingresos económicos, educación, salud, agua, electricidad, carreteras, tierra para producir, entorno natural saludable y otros recursos que facilitan la existencia de una familia.

Recibido: noviembre 2009/ Aceptado y versión final: enero 2010.

* Consultor independiente, Lic. en Economía, Diplomado en Objetivos de Desarrollo del Milenio con Énfasis en Análisis Sociodemográfico.

** Consultora independiente, Dasonónoma, ESNACIFOR, Diplomada en Objetivos de Desarrollo del Milenio con Énfasis en Análisis Socio demográfico. 
En el presente documento se presentan los diferentes hallazgos encontrados en los respectivos lugares investigados, los relatos y testimonios de los actores beneficiados en dicho proceso.

Se espera que el lector o lectora conozca los proyectos de inversión con fondos de ERP, analice los éxitos y fracasos de los mismos de manera que se conviertan en información oportuna para tomar decisiones en beneficio de la población intibucana y de San Isidro.

\section{JUSTIFICACIÓN}

Al aprobar la Declaración del Milenio en el año 2000, el compromiso de la comunidad internacional fue "no escatimaremos esfuerzos para liberar a nuestros semejantes, hombres, mujeres y niños de las condiciones abyectas y deshumanizadoras de la pobreza extrema". En este momento ya hemos recorrido nueve años desde que se comenzó con dicho proceso y la fecha límite se aproxima el año 2015, en el cual deberían lograrse los Objetivos de Desarrollo del Milenio, estos objetivos envuelven las aspiraciones de desarrollo del mundo en su conjunto. Pero no son sólo objetivos de desarrollo, representan valores y derechos humanos universalmente aceptados como la lucha contra el hambre, el derecho a la educación básica, el derecho a la salud y responsabilidad frente a las generaciones futuras.

La investigación tiene como propósitos describir el impacto obtenido producto de la inversión de los fondos de la Estrategia de Reducción de la Pobreza en los municipios de San Isidro e Intibucá, los que serán de considerable utilidad para los tomadores de decisiones en materia de fondos destinados a la reducción de la pobreza, así mismo contribuir aportando algunas recomendaciones fundamentales para que estos fondos mejoren en realidad las condiciones de vida de las familias más excluidas o los que viven en pobreza o extrema pobreza.

Aportar elementos reales de los propios beneficiarios, constatar sus verdaderos cambios en materia de mejoramiento de calidad de vida es uno de los aportes que pretende la investigación así mismo se podrá hacer alguna comparación de aquellos proyectos desarrollados que han generado verdaderos resultados positivos en un mejor nivel de vida de las familias y personas y describir de forma simple la forma que hizo posible el éxito o el fracaso de los proyectos.

\section{PLANTEAMIENTO DEL PROBLEMA}

San Isidro e Intibucá son municipios que han venido ejecutando proyectos desde que se comenzó a desembolsar los fondos a las municipalidades, provenientes de la Estrategia de Reducción de Pobreza (ERP) impulsada por el gobierno de Honduras como una Política de Estado.

Con el propósito de determinar su impacto se plantea el desarrollo de un estudio que permita observar in situ cada una de las acciones iniciadas al respecto a fin de determinar el impacto de la inversión de los fondos de la ERP en dichos municipios y como estos proyectos han mejorado las condiciones de vida de la población residentes en estas comunidades.

Es importante determinar si cada uno de los logros alcanzados una vez finalizados los proyectos, ha llenado las expectativas de los pobladores y si han tenido un impacto favorable en relación a la reducción de la pobreza.

\section{OBJETIVOS}

\subsection{Objetivo General:}

- Conocer el nivel de percepción de los beneficiarios con respecto a la Estrategia de Reducción de la Pobreza, tomando como base sus proyectos ejecutados desde que se comenzó con el programa, así mismo determinar el impacto obtenido producto de dicha intervención.

\subsection{Objetivos Específicos:}

- Enlistar los resultados positivos generados como producto de la inversión de los recursos provenientes de la Estrategia de la Reducción de la Pobreza (ERP) en los municipios de San Isidro e Intibucá. 
- Determinar los factores que han hecho posible el logro o fracaso de la inversión de los recursos en Intibucá y San Isidro.

- Enumerar las recomendaciones que los beneficiarios plantean para que los resultados en las futuras intervenciones sean más positivos.

- Comparar logros entre municipios y analizar sus resultados.

\section{METODOLOGÍA}

El proceso metodológico para la investigación comprende las fases de una revisión bibliográfica e información que se encuentra en la base de datos del Censo de Población y Vivienda del 2001 y la observación e indagación con actores involucrados en la planificación y ejecución de proyectos financiados con fondos de ERP.

Las fases de este proceso son las siguientes:

\subsection{Recopilación de información}

Se procedió a la recopilación de toda la información factible respecto al tema, para ello se accedió a diferentes tipos de fuentes como son: base de datos del CPV 2001; revisión de informes en las municipalidades con previa autorización de las autoridades; entrevistas con actores involucrados en la planificación y ejecución de proyectos.

\subsection{Sistematización de la Información}

Se revisó la documentación recolectada para sintetizar información. Se utilizó el Programa REDATAM y la base de datos del Censo Poblacional y Vivienda del 2001, así mismo herramientas de Microsoft Word y Excel.

\subsection{Análisis de la información}

$\mathrm{Al}$ analizar, evaluar e interpretar la información según la bibliografía revisada. Basado en la información analizada se obtuvo los resultados (datos) con lo cual desarrollo la investigación, estableciendo luego las conclusiones generales de la presente monografía basado en los objetivos propuestos.

\section{CONTEXTO DEL ESTUDIO}

\subsection{Datos Generales}

El departamento de Intibucá se encuentra en la región occidental del país; cuenta con 17 municipios y 127 aldeas. Según proyecciones realizadas por el INE, en el 2009 la población del departamento es de 226,227 personas de las cuales $49 \%$ son hombres y $51 \%$ mujeres ubicados en 34,716 viviendas.

El municipio de Intibucá está conformado por 20 aldeas y un total de 129 comunidades, el área urbana comprende 12 barrios. Para el 2009 según proyecciones del INE, Intibucá contaba con una población de 52,572 de los cuales 25,334 son hombres y 27,238 mujeres. Un $75 \%$ de la población del municipio sabe leer y escribir, se cuenta con centros educativos desde el nivel de prebásico hasta nivel universitario, así mismo hay oportunidad de educación bilingüe. La actividad agrícola es la principal fuente de ingresos en el municipio.

San Isidro está conformado por el casco urbano 2 aldeas y 3 caseríos, estas comunidades están distribuidas en la parte media del municipio y la comunidad que cuenta con mayor número de habitantes es Macuelizo. Proyecciones realizadas por el INE en el 2009 registran 4,140 personas de las cuales $52 \%$ son hombres y $48 \%$ mujeres. Un $68 \%$ de la población del municipio sabe leer y escribir, se cuenta con centros educativos desde el nivel de pre-básico hasta nivel básico, la oportunidad de los siguientes niveles la ofrecen municipios vecinos de Jesús de Otoro, Siguatepeque y la Esperanza. La agricultura es la principal fuente de ingresos en el municipio.

\subsection{La Estrategia de Reducción de la Pobreza (ERP)}

La Estrategia de Reducción de la Pobreza es un acuerdo ejecutivo que considera las fases, la tipología de proyectos y requisitos para acceso al fondo de reducción de la pobreza en Honduras.

El artículo especifica que los fondos: "serán destinados única y exclusivamente al financiamiento de los programas y proyectos municipales de inversión, focalizados y priorizados en cada circunscripción municipal 
por sus respectivas corporaciones, en amplios procesos de consulta con la comunidad."

En aquellos casos que las corporaciones así lo determinen, podrá utilizarse la instancia de las mancomunidades legalmente constituidas y certificadas, para cualquiera de las etapas del ciclo de proyectos. Así mismo, las Corporaciones Municipales tendrán en consideración, según sea el caso, las Estrategias Regionales para la Reducción de la Pobreza formuladas al efecto y los espacios regionales constituidos para los propósitos de ejecutar la ERP en ámbitos espaciales diferentes al de los municipios o de las mancomunidades.

Así mismo el acuerdo ejecutivo enfatiza que la formulación de proyectos debe estar enmarcada en los planes estratégicos de cada municipio, o en su caso de los planes de ordenamiento territorial, siempre y cuando sean proyectos socializados con la comunidad o presentados por las mismas. Da mucha importancia a que los que formulen proyectos para beneficiarios directos, pertenezcan a sectores vulnerables como la niñez, adolescencia, mujeres, grupos étnicos, etc.

En el artículo 7 se presenta la creación de una cuenta única para manejar el fondo de ERP con una contabilidad separada en cada municipalidad. El artículo 9 estipula las condiciones y procedimientos para obtener los desembolsos según los montos de inversión. En los artículos 10 y 11 encontramos el papel que juega una auditoria social como vigilante de la transparencia de las gestiones municipales y en la ejecución de los proyectos. Seguidamente se encuentran disposiciones de herramientas y procedimientos que deben aplicarse para invertir con fondos de ERP. (En el anexo 1 se encuentra al detalle el acuerdo ejecutivo de la estrategia de reducción de la pobreza en Honduras).

\subsection{Como se deben invertir los fondos de ERP en Honduras}

De acuerdo a lo establecido en el Reglamento el artículo 22 de las Disposiciones Presupuestarias contenidas en el Presupuesto General de Ingresos y Egresos de la República, para la operacionalización de los Recursos de la Estrategia de Reducción de la Pobreza (ERP), los recursos serán destinado única y exclusivamente al financiamiento de los proyectos municipales de inversión, focalizados y priorizados en cada municipalidad por sus respectivas corporaciones, en amplios procesos de consulta con la comunidad y las organizaciones locales de la sociedad civil.

En consideración a que el origen de los recursos es derivado de los fondos de alivio a la deuda, los beneficiarios deberán ser personas de escasos recursos, incluidos en el segmento poblacional por debajo de la línea de pobreza y particularmente, de la línea de pobreza extrema.

Por la importancia que tienen la apertura comercial actual y sus exigencias de productividad, competitividad, sellos de calidad, verdes y éticos de nivel internacional, se debe estimular todas las formas de cooperación interempresarial y la asociatividad. Así mismo, se deberá promover cadenas productivas y cadenas de valor que permitan además de elevar el valor agregado de los bienes y servicios incrementar los niveles de producción, productividad y competitividad nacional, regional e internacional.

En las zonas rurales particularmente, se deberá enfatizar en realizar procesos en el marco de un desarrollo económico local, desarrollo rural empresarial sostenible y de unidades de producción concentrada.

Los proyectos formulados con estos propósitos deberán buscar integrar la rentabilidad económica con la equidad social y la compatibilidad ambiental.

Los proyectos implementados por la ERP se dividen en tres ejes: eje productivo, el eje social y el eje de fortalecimiento institucional.

\section{PROYECTOS DE INVERSIÓN DE ERP EN MUNI- CIPIO DE INTIBUCÁ}

En el municipio de Intibucá la implementación de proyectos con fondos de la ERP consideró los tres ejes anteriormente mencionados, el cuadro No. 1 presenta la cartera de 11 proyectos aprobados en el 2007 y desembolsados a la municipalidad de Intibucá en el año 2008. 
Cuadro No. 1. Cartera de Proyectos de Municipio de Intibucá Aprobados por ERP en el año 2007.

\begin{tabular}{|c|c|c|c|c|}
\hline Código & Nombre del Proyecto & Eje Productivo & Eje Social & Eje Institucional \\
\hline $1006-001$ & Fortalecimiento y mejoramiento de envasados hortofrutícolas. & $137,275.00$ & & \\
\hline $1006-002$ & Mujeres Lencas al Éxito. & $463,265.18$ & & \\
\hline $1006-003$ & Suministros materia prima Tejidos Alianza. & $52,000.00$ & & \\
\hline $1006-004$ & Producción Hortícola del Norte de Intibucá. & $649,982.50$ & & \\
\hline $1006-005$ & Establecimiento de Fincas de Café. & $157,477.32$ & & \\
\hline $1006-006$ & Construcción de Modulo SAT. & & $195,392.11$ & \\
\hline $1006-007$ & Ampliación Escuela de Aplicación Honduras. & & $178,046.44$ & \\
\hline $1006-008$ & Perforación de Pozos y Tanques de Almacenamiento de Agua. & & $365,931.55$ & \\
\hline $1006-009$ & Techo de la Biblioteca Llano de la Virgen. & & $65,024.00$ & \\
\hline $1006-0010$ & Mejoramiento de Red de Distribución de Agua potable de Intibucá. & & $125,008.87$ & \\
\hline \multirow[t]{2}{*}{$1006-0011$} & Fortalecimiento Institucional de Alcaldía de Intibucá. & & & $265,551.03$ \\
\hline & TOTAL & $1,460,000.00$ & $929,402.97$ & $265,551.03$ \\
\hline
\end{tabular}

Fuente: Elaboración propia basada en información recopilada en Municipalidad de Intibucá.

El monto total de los proyectos fue de 2, 654,954.00 se invirtió el $55 \%$ en el eje productivo incluyendo 3 proyectos donde las beneficiarias directas fueron mujeres dedicadas a microempresas de envasados, tejidos y cultivo de flores, 2 proyectos de producción en el área de hortalizas y café, un 35\% en el eje social: 3 proyectos dirigidos al sector educativo y 2 en el sector salud específicamente de agua potable.

El 10\% restante se invirtió en el eje institucional. Es importante mencionar que el primer desembolso recibido fue de 1, 061,981.60 lo que representó el 40\% del total de fondos asignados
En el 2008 fue aprobada una cartera de 11 proyectos que fueron desembolsados a la municipalidad de Intibucá en el año 2009 (Ver cuadro No. 2)

Según el monto total de 5, 210,000.00 se invirtió el $58 \%$ en el eje productivo con un sólo proyecto de entrega de insumos. Y el $42 \%$ en el eje social que comprende 5 módulos educativos, construcción de 2 puentes, techado de varias viviendas y reparación de calles.

De manera muy general se detalla la información obtenida en los principales proyectos ejecutados en el municipio desde el punto de vistas de los actores involucrados en cada investigación (Cuadro No. 3).

\section{Cuadro No. 2. Cartera de Proyectos de Municipio de Intibucá Aprobados en el año 2008}

\begin{tabular}{|c|c|c|c|}
\hline Código & Nombre del Proyecto & Eje Productivo & Eje Social \\
\hline $1006-001$ & Módulo Manazapa Centro & & $255,870.00$ \\
\hline $1006-002$ & Módulo Mercedes Manazapa & & $255,870.00$ \\
\hline $1006-003$ & Módulo el Naranjo & & $255,870.00$ \\
\hline $1006-004$ & Módulo en San Antonio. & & $255,870.00$ \\
\hline $1006-005$ & Módulo para Discapacitados & & $255,870.00$ \\
\hline $1006-006$ & Puente en Zilimania & & $214,788.00$ \\
\hline $1006-007$ & Puente en Quiaterique & & $214,788.00$ \\
\hline $1006-008$ & Techado del Centro Básico de Chiligatoro & & $214,788.00$ \\
\hline 1006-009 & Reparación de Calles del Casco Urbano & & $200,000.00$ \\
\hline $1006-0010$ & Techos de varias viviendas & & $65,004.45$ \\
\hline \multirow[t]{2}{*}{$1006-0011$} & Compra de insumos agrícolas. & $3,021,281.55$ & \\
\hline & TOTAL & $3,021,281.55$ & $2,188,718.45$ \\
\hline
\end{tabular}

Fuente: Elaboración propia basada en información recopilada en Municipalidad de Intibucá 
Cuadro No. 3. Municipio de Intibucá: Principales proyectos ejecutados con fondos de la ERP

\begin{tabular}{|c|c|c|c|}
\hline Nombre del Proyectos & Inversión & Comunidad Beneficiada & Percepción de los actores y beneficiarios \\
\hline $\begin{array}{l}\text { Aula SAT: Escuela de Apli- } \\
\text { cación Honduras }\end{array}$ & Lps.195, 392.11 & $\begin{array}{l}\text { Comunidad Santa Catarina } \\
\text { Beneficiarios directos } 111 \\
\text { alumnos de los cuales son } 98 \\
\text { niñas y } 13 \text { varones }\end{array}$ & $\begin{array}{l}\text { El mayor impacto se ha visto en los alumnos de } 2^{\text {do }} \text { hasta } 6^{\text {to }} \text { grado ya } \\
\text { que ahora manejan varios programas de la computadora y en un área } \\
\text { rural eso es un gran logro." "Los alumnos han reforzado sus cono- } \\
\text { cimientos al entrar en el mundo de la computadora. (Nelsy Martínez, } \\
\text { docente interina) } \\
\text { Mis hijas ya podían acceder a tres programas y se levantan felices } \\
\text { cuando tienen clases en la computadora (María Lourdes Vásquez, } \\
\text { madre de familia). } \\
\text { El proyecto lleva } 7 \text { meses de ejecución una vez que se logró la donación } \\
\text { de las computadoras por parte de Save The Children. }\end{array}$ \\
\hline $\begin{array}{l}\text { Reparación del techo y piso } \\
\text { de un módulo de la Escuela } \\
\text { de Aplicación República de } \\
\text { Honduras }\end{array}$ & Lps.178,046.44 & $\begin{array}{l}\text { Comunidad Santa Catarina } \\
\text { Beneficiarios directos } 111 \\
\text { alumnos de los cuales son } 98 \\
\text { niñas y } 13 \text { varones }\end{array}$ & $\begin{array}{l}\text { "Si no hubieran estado las columnas que sostienen el techo reparado } \\
\text { del módulo en el temblor el techo se hubiera caído por completo" (Sr. } \\
\text { Indalecio Murillo, Director de la Escuela ) }\end{array}$ \\
\hline Módulo para Discapacitados & Lps. $255,870.00$ & $\begin{array}{l}\text { Los beneficiados lo conforman } \\
\text { aproximadamente el } 12 \% \text { de la } \\
\text { población total de los habitan- } \\
\text { tes en el municipio de Intibucá. }\end{array}$ & $\begin{array}{l}\text { El módulo brindará la oportunidad de entrenar en algunos oficios a } \\
\text { los discapacitados que lo deseen, brindando así la oportunidad de auto } \\
\text { emplearse y obtener ingresos para sus familias (Sr. Nicolás Márquez y la } \\
\text { Srta. Suyapa Meza Sánchez fungen como Asesores de la Organización } \\
\text { de Discapacitados a nivel del municipio de Intibucá) }\end{array}$ \\
\hline Mujeres Lencas al Éxito & Lps.463, 265.18 & Comunidad de Chiligatoro & $\begin{array}{l}\text { Nosotras nos dedicamos al cultivo del clavel y somos } 12 \text { socias, el } \\
\text { tanque de almacenamiento de agua para utilizar en el sistema de riego, } \\
\text { así como el plástico para preparar los viveros, será de gran beneficio } \\
\text { en nuestro trabajo } \\
\text { (Sra. Franca González Sánchez) }\end{array}$ \\
\hline $\begin{array}{l}\text { Compra de Insumos Agrí- } \\
\text { colas }\end{array}$ & Lps 3, 021,281.55 & $\begin{array}{l}\text { El total de beneficiados directos } \\
\text { es de } 4,042 \text { productores de la } \\
\text { Red Hortofrutícola del departa- } \\
\text { mento de Intibucá }\end{array}$ & $\begin{array}{l}\text { Todos los que trabajamos el cultivo de maíz, papa, repollo, lechuga } \\
\text { y coliflor, seremos muy beneficiados por el proyecto esto aporta } \\
\text { positivamente en nuestra economía familiar (productor beneficiado) }\end{array}$ \\
\hline
\end{tabular}

Fuente: Elaboración propia

\subsection{Factores positivos y negativos que inciden en la} implementación de proyectos con fondos de la ERP.

\section{Factores Positivos}

Escuela de Aplicación República de Honduras

- El Director de la escuela funge como regidor de la municipalidad

- La capacidad de respuesta de la comunidad ante el desarrollo de la escuela.

- La priorización de la municipalidad de ejecutar proyectos en el área rural del municipio.

Módulo de los Discapacitados

- La organización de los discapacitados en el municipio, sin tener local y haciendo reuniones en el parque.

- La persistencia de dar a conocer sus demandas en los cabildos abiertos de la municipalidad.
- El apoyo fuerte y constante de la Asociación Intibucana de Mujeres "Las Hormigas" quienes les han capacitado para incidir en las autoridades locales y para gestionar proyectos.

Proyecto de Insumos Agrícolas

- La experiencia de gestión de proyectos de organizaciones campesinas en la zona.

- La anuencia de la Corporación Municipal

- El acompañamiento de la Red Hortofrutícola a la municipalidad de Intibucá en todo el proceso de gestión y ejecución del proyecto.

- La perseverancia en el proceso de gestión.

- Una planificación de siembra municipal involucrando varias organizaciones a la vez.

- El objetivo del proyecto es una necesidad sentida por los productores. 
De manera general mencionamos que un factor de éxito ha sido la anuencia de las autoridades municipales en coordinar con otros actores la formulación e implementación de proyectos. Otro factor es la puesta en práctica de instrumentos normativos como ser la Ley de Contratación del Estado, en el sentido de hacer las compras por medio de licitación pública para demostrar transparencia en el proceso y obtener los mejores precios de insumos.

\section{Factores Negativos}

En cuanto a los factores negativos se puede mencionar los siguientes:

- La enorme demanda de recursos provenientes de la ERP por parte de las comunidades ha dificultado beneficiar a todas las comunidades del municipio.

- Geográficamente el municipio es muy disperso lo que dificulta un monitoreo efectivo, tanto en la formulación como en la ejecución de los proyectos.

- La forma de desembolsar los recursos de la ERP en porcentajes por parte del gobierno central ocasionó atrasos en la implementación de los proyectos priorizados; tal es el caso que para proyectos de producción basados en un plan de siembra, si no se tienen todos los insumos necesarios la planificación ya no tiene éxito. Se desperdician las condiciones climáticas que son un factor indispensable para cualquier cultivo.

- La municipalidad no contaba con el recurso humano calificado para realizar los estudios de prefactibilidad y factibilidad de proyectos.

- Por parte del Gobierno Central no habían mecanismos claros de implementación del fondo de la Estrategia de Reducción de la Pobreza.

- El plan estratégico municipal no respondía en el sector productivo y otras áreas de tipo social que las comunidades demandaban, ya que estaba más orientado a la infraestructura física.

- La inexistencia de organismos de control social como las comisiones de transparencia y el comisionado municipal que son requisitos exigidos por el reglamento del fondo de la ERP. Tales organismos generan confianza en el manejo de los recursos.

\section{Perspectivas de los beneficiados.}

- La priorización de proyectos debe ser primero para la zona rural.

- Dar más participación en los cabildos abiertos para presentar ideas de proyectos.

- Mayor calidad de información de presupuestos.

- Mucha transparencia en la presentación del resultado de las inversiones.

- Proceso de desembolsos más eficaces.

\subsection{Enfoque de Género en los proyectos ERP en el Municipio de Intibucá}

El enfoque de género en los proyectos que se ejecutaron con fondos de ERP se visualiza como la cantidad de mujeres beneficiarias, además de la aceptación de propuestas de proyectos dirigidas a empresas de mujeres.

El cuadro No. 1 presenta los diferentes proyectos visitados y la cantidad de mujeres beneficiarias por cada uno. Sin embargo, se puede agregar que la participación de mujeres dependía del tipo de proyecto en el que se involucran, tal es el caso de microempresas de flores, de hilados o de envasados Cuadro No. 4.

En relación a la cantidad de mujeres beneficiadas el impacto se visualiza más cuando los proyectos son formulados e implementados por mujeres, asegurando

Cuadro No. 4. Porcentaje de participación de Mujeres en Proyectos Financiados por ERP

\begin{tabular}{|c|c|c|c|c|}
\hline \multirow{2}{*}{ Año } & \multirow{2}{*}{ Proyectos } & \multicolumn{2}{|c|}{ Beneficiarias } & \multirow{2}{*}{$\begin{array}{l}\% \text { de Par- } \\
\text { ticipación } \\
\text { Mujer }\end{array}$} \\
\hline & & Mujeres & Hombres & \\
\hline \multirow[t]{4}{*}{2007} & Mujeres Lencas al éxito & 12 & 0 & $100 \%$ \\
\hline & $\begin{array}{l}\text { Fortalecimiento Y mejo- } \\
\text { ramiento de envasados } \\
\text { hortofrutícolas }\end{array}$ & 255 & 0 & $100 \%$ \\
\hline & Módulo SAT & 98 niñas & 13 niños & 88.3 \\
\hline & Módulo discapacitados & $51 \%$ & $49 \%$ & $51 \%$ \\
\hline 2008 & Insumos Agrícolas & 775 & 1932 & $28.6 \%$ \\
\hline
\end{tabular}

Fuente: Elaboración propia según datos recolectados en las visitas. 
que sus ingresos repercuten de manera directa en los dependientes de la pareja, tal es el caso de la Asociación de Mujeres Artesanas de Intibucá (AMIR) que la conforman 267 mujeres aglutinadas en 17 grupos.

\section{PROYECTOS DE INVERSIÓN DE ERP EN MU- NICIPIO DE SAN ISIDRO}

El municipio de San Isidro fue beneficiado a partir del 2006 con proyectos de fondos de la ERP, al igual que en el municipio de Intibucá se consideraron los tres ejes contemplados en la tipología de proyectos de la ERP.

El cuadro No. 5 presenta la cartera de 5 proyectos que en el año 2006 fueron aprobados, pero desembolsados a la municipalidad de San Isidro en el año 2007. Según el monto total de 2,424,982.43 se invirtió el 54\% en el eje productivo; el $35.8 \%$ en el eje social que comprende reparación del CESAMO, letrinización y mejoramiento de viviendas; y el $10.2 \%$ restante en el eje institucional.

El cuadro No. 6 presenta la cartera de 12 proyectos del 2007 que le fueron aprobados y desembolsados a la municipalidad de San Isidro en el año 2008. Según el monto total de 2, 386,732.93 se invirtió el 27.9\% en el eje productivo (café y huertos familiares); el $65.4 \%$ en el eje social que comprende electrificación y reparación de centros educativos; y el $6.7 \%$ restante en el eje institucional y el cuadro No. 7 muestra los principales proyectos ejecutados con fondos ERP.

Cuadro No. 5. Cartera de Proyectos de Municipio de San Isidro Aprobados en el año 2006

\begin{tabular}{|c|c|c|c|c|}
\hline No & Nombre del Proyecto & Eje Productivo & Eje Social & $\begin{array}{l}\text { Eje Institu- } \\
\text { cional }\end{array}$ \\
\hline 1 & Adquisición de Equipo tecnológico formación local y comunitaria & & & $247,498, .23$ \\
\hline 2 & Caja Rural San Isidro Centro & $1,311,240.40$ & & \\
\hline 3 & Mejoramiento y Ampliación de CESAMO & & $329,645.01$ & \\
\hline 4 & Mejoramiento de Vivienda & & $375,000.12$ & \\
\hline \multirow[t]{2}{*}{5} & Proyecto de Letrinización & & $161,598.67$ & \\
\hline & TOTAL & $1,311,240.40$ & $866,243.80$ & $247,498.23$ \\
\hline
\end{tabular}

Fuente: Elaboración propia basada en información recopilada en Municipalidad de San Isidro.

Cuadro No. 6. Cartera de Proyectos de Municipio de San Isidro Aprobados en el año 2007.

\begin{tabular}{|c|c|c|c|c|}
\hline No & Nombre del Proyecto & Eje Productivo & Eje Social & Eje Institucional \\
\hline 1 & Cultivo de café & $350,000.00$ & & \\
\hline 2 & Construcción de Proyecto Electricidad Macuelizo & & $695,000.00$ & \\
\hline 3 & Formación de Huertos familiares & $316,240.25$ & & \\
\hline 4 & Bajadas domiciliarias de energía eléctrica & & $295,000.00$ & \\
\hline 5 & Construcción de Escuela Miguel Ángel Gámez el (Borbollón). & & $471,243.80$ & \\
\hline 6 & Reparación de Techo Jardín ( Nuevo Amanecer) & & $100,000.00$ & \\
\hline 7 & Capacitación Normas Parlamentarias, organización a patronatos & & & $40,856.38$ \\
\hline 8 & Dotación de equipo tecnológico a la comisión Transparencia y C.M & & & $58,549, .73$ \\
\hline 9 & Capacitación a personal Administrativo de la Municipalidad & & & $28,942.67$ \\
\hline 10 & Fortalecimiento a procesos & & & $40,000.00$ \\
\hline 11 & Capacitación para Auditorias Sociales por el T.S.C & & & $49,449.83$ \\
\hline \multirow[t]{2}{*}{12} & Supervisión de Proyectos & & & $4,949.97$ \\
\hline & TOTAL & $666,240.25$ & $1,561,243.80$ & $159,248.88$ \\
\hline
\end{tabular}

Fuente: Elaboración propia basada en información recopilada en Municipalidad de San Isidro. 
Tabla No. 7. Municipio de San Isidro: Principales proyectos ejecutados con fondos de la ERP

\begin{tabular}{|c|c|c|c|}
\hline Nombre del Proyectos & Inversión & $\begin{array}{l}\text { Comunidad } \\
\text { Beneficiada }\end{array}$ & Percepción de los actores y beneficiarios \\
\hline $\begin{array}{l}\text { Proyecto Caja Rural } \\
\text { Unión y Esfuerzo }\end{array}$ & $1,311,220.46$ & San Isidro & $\begin{array}{l}\text { Actualmente la caja rural tiene un déficit del fondo capital semilla de Lps } \\
92,859.20 \text { lo que demuestra una débil capacidad administrativa que los } \\
\text { puede llevar al fracaso total del fondo. } \\
\text { Actualmente nadie ha pagado capital, ni intereses, por lo que el fondo } \\
\text { no ha podido crecer, esto podrá verse a finales de Marzo de } 2010\end{array}$ \\
\hline $\begin{array}{l}\text { Mejoramiento y Amplia- } \\
\text { ción de CESAMO. }\end{array}$ & $329,645.40$ & San Isidro & $\begin{array}{l}\text { La ampliación del CESAMO permitirá trabajar con mayor comodidad, } \\
\text { dado que diariamente se atienden } 30 \text { personas. } \\
\text { Una de las piezas será utilizada para almacén y la otra para nebulizaciones } \\
\text { y esterilización (Enfermera del CESAMO) }\end{array}$ \\
\hline $\begin{array}{l}\text { Mejoramiento de vi- } \\
\text { viendas }\end{array}$ & $375,000.00$ & $\begin{array}{l}190 \text { familias de la comu- } \\
\text { nidad de San Isidro }\end{array}$ & $\begin{array}{l}\text { Muchas de las familias invirtieron el material en el mejoramiento de sus } \\
\text { viviendas, sin embargo, un número muy pequeño de familias vendieron } \\
\text { sus materiales para satisfacer sus necesidades básicas de alimentación. } \\
\text { (Residente San Isidro) }\end{array}$ \\
\hline $\begin{array}{l}\text { Adquisición de equipo } \\
\text { tecnológico formación } \\
\text { local y comunitaria }\end{array}$ & $247,498, .23$ & $\begin{array}{l}\text { Municipalidad de San } \\
\text { Isidro }\end{array}$ & $\begin{array}{l}\text { Este proyecto ha ayudado a la municipalidad a que haya modernizado } \\
\text { su equipo de cómputo municipal usado en los distintos departamentos } \\
\text { que brindan un servicio más eficiente y oportuno a la ciudadanía, pero } \\
\text { presentan debilidad en el mantenimiento del mismo }\end{array}$ \\
\hline Letrinización & $161,598.67$ & San Isidro & $\begin{array}{l}\text { Este proyecto ha permitido mejorar las condiciones de saneamiento } \\
\text { básico de la comunidad (Regidor municipal) }\end{array}$ \\
\hline
\end{tabular}

8.2 Factores positivos y negativos que inciden en la implementación de proyectos con fondos de la ERP

\section{Factores Positivos}

De manera general se visualiza que los factores de éxito en cada uno de los proyectos ejecutados en el municipio de San Isidro, se debieron a:

- El alto grado de motivación de los beneficiarios.

- La participación de la comunidad en la propuesta de las ideas de proyecto.

- El área geográfica del municipio está bien concentrada, lo que facilita el monitoreo de cada una de las etapas que comprende la ejecución de un proyecto.

- La comunicación permanente de las autoridades que forman la corporación municipal.

- El desarrollo de mecanismos de participación como cabildos abiertos y reuniones de corporación en la que participan los líderes de las diferentes comunidades para exponer sus ideas de proyectos.

- El empoderamiento de la metodología que facilita la implementación de los proyectos contenida en la Estrategia de Reducción de la Pobreza.
- El uso del plan estratégico municipal para priorizar las necesidades más sentidas de cada una de las comunidades.

Los factores de fracaso para el municipio de San Isidro son similares a los presentados en el municipio de Intibucá, a excepción de los dos primeros que se relacionan al área geográfica.

\subsection{Perspectivas de los beneficiados.}

- Se considera que el proyecto de mayor impacto fue el proyecto de viviendas el cual benefició a muchas familias que tenían techos de paja, pisos de tierra.

- La instalación de luz eléctrica ha generado mejor condición de vida, ya que se gasta menos leña, hay menos contaminación y se puede hacer negocio por medio de venta de productos alimenticios que necesitan refrigeración para su conservación como carnes etc.

- Los fondos para la ERP, deben ser invertidos en producción ya que se necesita para el consumo y la venta y así se puede vivir mejor.

- Ha habido un gran beneficio a las familias con los fondos de la ERP, sin embargo, se reconoce que las 
necesidades de la gente son muchas y es muy difícil que salgan de la pobreza.

- A la gente no le gusta estar organizada, ya que en los grupos hay mucho egoísmo, eso contribuye a que se pierdan muchas veces las ayudas y en forma organizada las ideas se desarrollan en consenso y se les busca solución a los problemas comunitarios, esto hace que el desarrollo avance con mayor porcentaje debido a que hay mucho analfabetismo en la gente.

\subsection{Enfoque de Género en los proyectos ERP en el Municipio de San Isidro}

Según datos proporcionada por personal encargado de administrar la información de la Estrategia de la Reducción de la ERP de la municipalidad de San Isidro, registran que en todos los proyectos ejecutados con estos fondos totalizan 2,050 beneficiarios, de la cuales 1,050 son hombres y 1,000 son mujeres (Gráfico No.1).

De lo anterior se deduce que la participación de la mujer es menor en comparación con la de los hombres, por lo que aún en el siglo XXI, se continúa con esa desigualdad entre sexos principalmente en estos tiempos en que la mujer tiene una destacada participación en la

\section{Gráfico No. 1. Beneficiarios Directos ERP en el Municipio} de San Isidro.

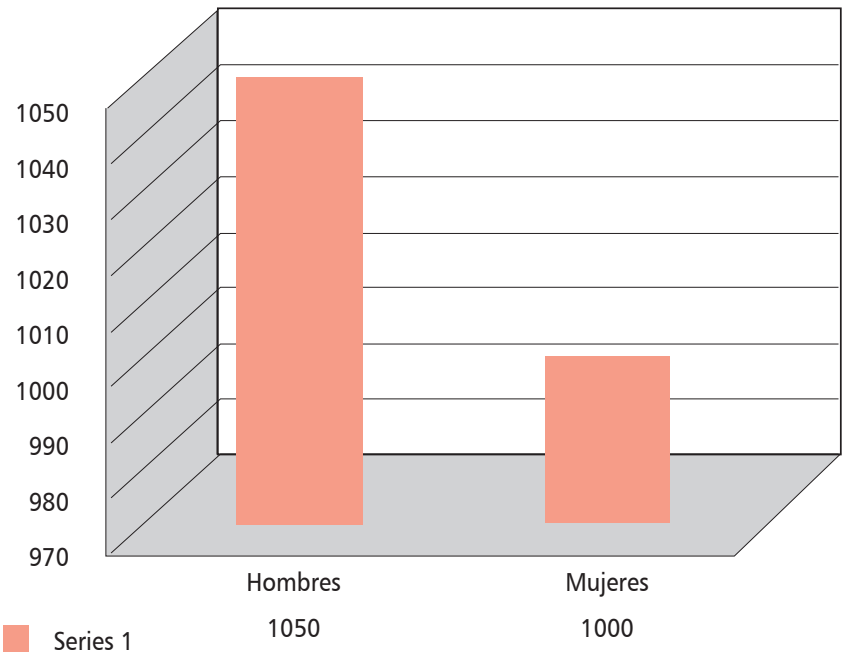

Fuente: Elaboración propia basada en información recopilada en la Municipalidad. generación de los ingresos familiares y otros campos del desarrollo comunitario y municipal.

\section{CONCLUSIONES}

- En ambos municipios las personas beneficiadas en cada uno de los proyectos demuestran un grado de satisfacción por haber recibido un beneficio, sin embargo, no cumple al $100 \%$ con sus expectativas, tal es el caso de los proyectos de mejoramiento de vivienda que no contemplaron todos los insumos básicos para un mejoramiento total.

- Actualmente las comunidades de los municipios de San Isidro e Intibucá, cuentan con los conocimientos mínimos para aprovechar los recursos que provienen de la Estrategia de Reducción de la Pobreza.

- El proceso desarrollado hasta el momento por cada municipalidad concerniente a la formulación y ejecución de proyectos con fondos de ERP, ha dejado un aprendizaje en los actores involucrados (comunidad, beneficiarios, corporaciones y $\mathrm{ONG}$ ) que facilitará las nuevas carteras de proyectos que se gestionen.

- Una vez emitido el acuerdo de la ERP la debilidad para acceder al fondo de forma inmediata en ambas municipalidades, fue la falta de capacidad instalada, especialmente el recurso humano calificado y los organismos de control social.

- Al comparar las carteras de proyectos priorizados por ambas municipalidades, encontramos que Intibucá priorizó la inversión de sus recursos en un 58\% en el eje productivo contra un $42 \%$ para el eje social; sin embargo, en San Isidro fue para el eje social en un $95.9 \%$ y el eje productivo en un $4.1 \%$ podemos concluir que esta situación se debió a los siguientes factores: a) Aspectos geográficos que limitaron la presentación de demandas de manera inmediata. b) La presentación de propuestas del eje productivo en Intibucá se coordinó a través de organizaciones productoras y comercializadoras del agro, en cambio en San Isidro se hizo de forma individual.

- La participación de la mujer en proyectos financiados con fondos de la ERP en Intibucá y San Isidro 
presentan diferencias significativas debido al grado de organización de la mujer intibucana. Por lo anterior se concluye que es necesario propiciar en San Isidro los espacios de participación de la mujer de manera organizada para impulsar nuevas formas de generación de ingresos para mejorar su nivel de vida.

- A pesar de las experiencias obtenidas por las corporaciones municipales en la implementación de la ERP, todavía se presentan debilidades en la liquidación y formulación de proyectos, situación que atrasa la gestión de nuevos recursos.

\section{BIBLIOGRAFÍA}

- Cámara de comercio de comercio Intibucá.

- INE, Anuario Estadístico 2006. Aspectos Demográficos paginas 77-80.

- INE, Anuario Estadístico 2006. Aspectos Sociales, Salud, paginas 187-189.

- INE, Censo Poblacional y Vivienda, 2001.

- Municipalidad de San Isidro.

- Municipalidad de Intibucá.

- Red Hortofrutícola Intibucá, Informe de liquidación fondos de ERP, Agosto 2009. 